\title{
Acute Influences of Estrogen and Testosterone on Divergent and Convergent Thinking in Postmenopausal Women
}

\author{
Rosemarie Krug', Matthias Mölle', Christoph Dodt ${ }^{2}$, Horst L Fehm² and Jan Born*,' \\ 'Department of Neuroendocrinology, University of Lübeck, Germany; ${ }^{2}$ Department of Internal Medicine, University of Lübeck, Germany
}

\begin{abstract}
Previous studies indicated an enhanced capability of divergent creative thinking in young women during the ovulatory phase, which expressed itself also by an increased dimensional complexity of ongoing electroencephalographic (EEG) activity. Considering the enhanced plasma levels of estrogen and testosterone characterizing the ovulatory phase, we tested whether short-term administration of estrogen or testosterone in postmenopausal women with constantly low levels of gonadal steroids induces similar changes in divergent thinking. In two placebo-controlled cross-over studies, healthy postmenopausal women $(n=12$, in each study, mean age 58 years, range 47-65 years) were treated transdermally over 3 days with estrogen and testosterone, respectively, at doses inducing plasma hormone concentrations comparable with those observed in young women around ovulation. Capabilities of divergent thought and convergent analytical thought, performance on motor perseveration, and verbal memory were examined. EEG activity was recorded while subjects performed on tasks of thinking and during mental relaxation. Estrogen impaired divergent thinking $(p<0.0 \mathrm{I})$ and enhanced convergent thinking, motor perseveration, and memory for the initial word list ( $p<0.05$ for all tests). In parallel, EEG dimensional complexity was reduced $(p<0.05)$. Overall, these changes indicate an estrogen-induced shift from a 'divergent' towards a more 'convergent' mode of processing. However, overall less consistent, effects of testosterone were opposite to those of estrogen. It increased performance on some of the divergent thinking tasks $(p<0.05)$, and tended to increase EEG dimensional complexity during divergent thinking. Data indicate a differential sensitivity of modes of thinking to short-term treatment with estrogen and testosterone in postmenopausal women. Neuropsychopharmacology (2003) 28, I538-I545, advance online publication, I 4 May 2003; doi: I 0. I 038/sj.npp. I 300200
\end{abstract}

Keywords: estrogen; testosterone; thinking; working memory; EEG dimensional complexity

\section{INTRODUCTION}

There is growing evidence that cognitive functions and, in particular, aspects of working memory are subject to regulatory influences of systemic gonadal steroids. Support for this view derives from studies in young women, demonstrating specific changes in spatial and verbal abilities across the menstrual cycle (eg Hampson, 1990; Hausmann et al, 2000), as well as from studies in older postmenopausal women, which showed a rather consistent improvement in verbal memory function (immediate and delayed recall of words and stories) during estrogen replacement therapy (eg LeBlanc et al, 2001; Rice et al, 1997; Sherwin, 1999, 2002). A beneficial influence of estrogen on verbal memory was likewise suggested by studies comparing older women with high vs low basal levels of these hormones (Drake et al, 2000; Wolf and

*Correspondence: Dr J Born, Institut für Neuroendokrinologie, Universität zu Lübeck, Ratzeburger Allee 160, Haus 23a, D-23538 Lübeck, Germany, Tel: + 49 45I 500 364I, Fax + 49 45I 500 3640, E-mail: born@kfg.mu-luebeck.de

Received 26 September 2002; revised 08 January 2003; accepted 05 March 2003

Online publication: 27 March 2003 at http://www.acnp.org/citations/ Npp032703255/default.pdf
Kirschbaum, 2002). Estrogen therapy in postmenopausal women, in addition, was found to enhance functions of logical problem solving and verbal fluency (LeBlanc et al, 2001; Grodstein et al, 2000). For the latter function, distinct changes were also reported following testosterone replacement therapy (Sherwin, 1988; Regestein et al, 2001), although neuropsychological effects of androgen administration in women have overall rarely been investigated.

With respect to working memory functions in young women, we found particularly consistent changes for abilities of divergent creative thinking which are distinctly enhanced around the time of ovulation (Krug et al, 1994, 1996, 1999). Divergent thinking refers to the fluency and flexibility with which new representations become activated in working memory (Guilford, 1967; Guilford and Hoepfner, 1971). It is characterized by a destructured mode of mental processing that aims to activate as many mental representations as possible with only a weak associative connection to the task stimulus. Divergent thinking is usually contrasted with convergent thinking, which refers to an analytic mode of working memory operations focussing on a few strongly associated representations, the activation of which converges upon a final solution that is either right or wrong and whose mental representation is probably most clearly connected to all aspects of the task representation (Guilford, 
1967; Guilford and Hoepfner, 1971). Around ovulation, divergent creative abilities such as the production of fantasies and fluency of speech were found to be enhanced, whereas no such increase was found for convergent abilities of thinking, such as mental arithmetic (Brunel, 1991; Slob et al, 1996).

Based on Guilford's test battery of divergent thinking, our own studies confirmed a clear enhancement in divergent thinking performance during the ovulatory period as compared to performance at menses or the luteal phase, while performance on convergent tests of problem solving was not modulated by menstrual phase. Also women using contraceptives did not show a peak in creative performance at a time corresponding to the ovulatory period (Krug et al, 1994). Notably, in these studies the ovulatory enhancement in divergent thinking was paralleled by a marked enhancement in the dimensional complexity of electroencephalographic (EEG) activity (Krug et al, 1999). The dimensional complexity of the ongoing EEG is considered a correlate of the number of concurrently activated cortical neuron assemblies underlying the flow of cognitive representations (Lutzenberger et al, 1996; Mölle et al, 1999; Pritchard and Duke, 1995). Accordingly, divergent thinking requiring the quick and simultaneous activation of multiple cognitive representations is accompanied by increased EEG dimensional complexity in comparison with convergent thinking. Enhanced EEG complexity during the ovulatory phase would thus be expected to predispose cortical networks to more creative thought.

Considering the distinctly enhanced capability of divergent thinking in young women during the ovulatory phase characterized by enhanced plasma levels of estrogen and testosterone, in this study we tested whether a similar increase in divergent thinking could be induced in postmenopausal women by administration of either estrogen or testosterone alone. Using a transdermal route of administration, increases in plasma concentrations of these hormones were induced in postmenopausal women, which roughly resembled the short-term change observed in these hormonal levels in young, spontaneously cycling women. Aside from measures of divergent and convergent thinking and associated EEG activity, verbal memory and selfreported mood were assessed.

\section{MATERIALS AND METHODS}

\section{Subjects}

Healthy women aged between 47 and 65 years were solicited through advertisements. They were screened in a standardized interview and considered eligible if (i) menstrual bleeding had not occurred for at least 2 years and the woman had not experienced any perimenopausal symptoms since more than 1 year, (ii) they had not received estrogen replacement for at least 3 months before the experiments (five women had estrogen replacement before), and (iii) they were free of chronic or acute diseases, currently did not take any medication, and were nonsmokers. Analysis of serum hormone levels confirmed an estrogen concentration $<50 \mathrm{pg} / \mathrm{ml}$, a progesterone concentration $<2 \mathrm{ng} / \mathrm{ml}$, and a follicle stimulating hormone (FSH) concentration $>20 \mathrm{mlU} / \mathrm{ml}$ in each woman. Medical examination and history excluded psychiatric and neurological diseases (including premenstrual syndrome), cardiovascular risk factors, diabetes mellitus as well as acute infections. The sample with estrogen treatment included 12 women aged between 51 and 65 years (mean 58.6 years, mean body mass index \pm SEM: $\quad 23.4 \pm 0.5 \mathrm{~kg} / \mathrm{m}^{2}$ ) with a mean time of $9.1 \pm 1.4$ years after the last menstrual bleeding. The sample with testosterone treatment included 12 women aged between 47 and 65 years (mean 57.5 years, mean body mass index \pm SEM: $26.1 \pm 1.2 \mathrm{~kg} / \mathrm{m}^{2}$ ) with a mean time of $6.16 \pm 1.4$ years after the last menstrual bleeding. Subjects were paid and all gave written informed consent prior to participation. They were not aware of the particular study hypotheses examined. The study was approved by the local ethics committee.

\section{Design and Procedure}

The studies were performed according to a within-subject cross-over design. Each woman was tested on two experimental sessions, after administration of either placebo or an active hormone, that is, estrogen (Experiment I) and testosterone (Experiment II). Estrogen and testosterone were administered transdermally via a skin patch (Estraderm TTS100, Novartis Pharma Nuernberg, Germany; Testoderm 15, Ferring Arzneimittel Kiel, Germany), which was attached to the upper buttocks at $10.00 \mathrm{~h} 2$ days before the test session and delivered, respectively, about $0.1 \mathrm{mg}$ estradiol and $6 \mathrm{mg}$ testosterone daily. The patch was replaced by a new one at $10.00 \mathrm{~h}$ on the day of testing. Identical patches without the respective hormones were used for the placebo condition. Test sessions for a subject were separated by at least 3 weeks, and the order of treatment conditions was balanced across subjects. Subjects as well as the experimenter were blind against the actual treatment condition.

EEG during thinking. Experimental sessions started at $15.00 \mathrm{~h}$ and took about $2 \mathrm{~h}$. They took place in a soundattenuated room with the woman sitting in a reclining chair. EEG recordings were obtained during three mental conditions: while the women solved (i) a task of divergent thinking, (ii) a task of convergent thinking, and (iii) during mental relaxation. The order of these conditions was balanced across subjects, but was kept the same for a given subject across the two sessions. The tasks of divergent creative thought were taken from the section 'Consequences' of the Guilford test repertoire (Guilford, 1967), and were also used by Krug et al (1999). The woman was given a hypothetical situation (on one session, 'Due to a new finding people no longer need to eat....'; and on the other session, 'Imagine you were able to fly...') and was to think of as many unique consequences as possible. The tasks involving convergent thinking were chosen on the basis of the subtest 'arithmetical thinking' of the HamburgWechsler Intelligence Scale (HAWIE) (Wechsler, 1981) and required the logical operation of thought (eg 'Tina has as many sisters and brothers, but her brother John has twice as many sisters as brothers. How many sisters and brothers are there?'). During mental relaxation, subjects were to imagine a hypothetical situation ('You are lying on the beach, the sun is shining warmly, the waves are rolling softly'), and to 
relax. To enable EEG recording during thinking free of artifacts from movements of eyes and body, the tasks of divergent and convergent thinking included the following sequence of events that was repeated three times: (i) oral presentation of the task, (ii) followed by a 40-s time interval during which the woman had to think of an answer, (iii) and then a 60-s interval to answer the task in writing. For inducing divergent thinking, the story presented on each of the three repetitions remained the same and the subject was just asked to generate further new ideas on the second and third trials. For inducing convergent thinking three different tasks were presented, and the subjects were asked to write down not only the final solution but also partial solutions to subproblems.

EEG recordings were followed by four additional test conditions, with the order of these conditions balanced across subjects: (i) five tests of divergent thinking without EEG recordings, (ii) a test of motor perseveration, (iii) memory for words, and (iv) an assessment of mood.

Tests of divergent thinking without EEG. These tests were added in order to increase validity of the behavioral assessment of creative thinking. The four tests were adapted from Guilford (1967) and Wallach and Kogan (1969) and were considered by those authors to dissociate aspects of 'flexibility' and 'fluency' during creative thought. (1) 'Synonyms' is a test of fluency requiring the woman to produce as many synonyms as possible of a given word, for example, the adjective 'nice', within 2 min. (2) For the 'Pattern Meaning' test assessing flexibility, the woman is presented with abstract patterns (eg straight or curved lines) and is to write down all the things the pattern makes her think of, within 2 min. (3) 'Alternate Uses' is also a test of flexibility where the woman has to write down within 3 min as many unusual uses as possible for common things (eg a brick). (4) The 'Instances' test is a test of fluency concentrating on similarities among things. The woman is asked, for example, to write down all the round things she can think of within $2 \mathrm{~min}$. (5) The 'Planning' test assessing fluency requires the woman to specify within 3 min steps that make a given plan work, for example, a detailed plan for an excursion. The tests not only have proven valid indicators of divergent thinking capabilities (eg Bollinger, 1981) but were also found to distinctly discriminate thinking during different phases of the menstrual cycle (Krug et al, 1994). The tests were always given in the same order. Alternate versions of the tests were presented on the two sessions of an individual woman.

Motor perseveration. This test assesses tendencies of stereotyped motor behavior (Mittenecker, 1960; Schwibbe, 1985). The subject is confronted with a keyboard with nine red buttons randomly distributed over the board. The subject's task is to press the buttons with the order of button press responses being as 'chaotic' as possible. Button press responses were paced by a metronome $(64 / \mathrm{min})$, and a series of 243 responses was required. The test was controlled by a microprocessor determining a measure of entropy on the basis of the frequency and the sequential probability of each button pressed (Mittenecker, 1960). Since a high entropy value means a low tendency to perseverate, the reciprocal of this value was used to score motor perseveration. Enhanced motor perseveration is reliably found, for example, in mentally retarded persons and after intake of alcohol (Schwibbe, 1985). Also, persons with good capabilities of divergent thinking are assumed to show little motor perseveration.

Verbal memory. The immediate free recall of words from two lists, each containing 15 words, was assessed. Both lists contained one-syllable nouns, which were matched with regard to frequency and imagery. There were no obvious semantic relations among the words of the two lists. The words were presented by a tape recorder with a rate of 1 word per second. After each list a 60-s interval was given for verbal recall. Different words were used on the two experimental sessions for a subject. The number of words correctly recalled was used as performance measure.

Mood. Self-reported feelings of activation and mood were assessed by a standardized checklist of adjectives (Eigenschaftswörterliste - EWL, Janke and Debus, 1978). The EWL consists of 161 adjectives describing the actual state of mood on 15 dimensions such as 'activation', 'concentration', 'tiredness', 'anxiety', 'depression', 'self-reliance', etc. For each adjective, the woman indicated whether or not it accurately describes her actual feelings.

At the end of the session, after a 15-min resting period blood was sampled to determine estrogen, progesterone, testosterone, FSH, and luteinizing hormone (LH). Blood samples were immediately centrifuged at $4{ }^{\circ} \mathrm{C}$ and serum was stored at $-80^{\circ} \mathrm{C}$ until assay.

\section{Recordings and Apparatus}

The EEG and concomitant electrooculographic activity (EOG) was recorded continuously during the 40-s epochs of divergent and convergent thinking as well as during the corresponding epoch of mental relaxation. The EEG was recorded with nonpolarizable $\mathrm{Ag} / \mathrm{AgCl}$ electrodes (diameter $8 \mathrm{~mm}$, Sensomedics, The Netherlands) attached to left, midline, and right frontal (F3, Fz, F4), central (C3, Cz, C4), parietal $(\mathrm{P} 3, \mathrm{Pz}, \mathrm{P} 4)$, and left and right occipital $(\mathrm{O} 1, \mathrm{O} 2)$ scalp locations. Linked electrodes at the mastoids served as reference, and a ground electrode was attached to the forehead. Three additional electrodes were used to record vertical and horizontal eye movements. Interelectrode impedance was always $<5 \mathrm{k} \Omega$. EEG and EOG signals were amplified and filtered within a bandwidth of $0.16-35 \mathrm{~Hz}$ $(-3 \mathrm{~dB},-12 \mathrm{~dB} /$ octave) by a Nihon Koden electroencephalograph (Model 4400). A personal computer was used to acquire the signals via an ADC interface (CED 1401, Cambridge Electronic Design, UK) at a sampling rate of $100 \mathrm{~Hz}$. To minimize EOG artifacts, subjects were instructed to keep their eyes open and to fixate on a spot on the facing wall during EEG recordings.

\section{Data Reduction}

EEG analysis was conducted as described previously (Mölle et al, 1999). For each task of thinking a 20.48-s epoch free of large eye movements and other artifacts was selected within the 40-s interval of thinking, resulting in three epochs for 
each type of task. Correspondingly, three 20.48-s epochs of EEG recordings were selected during mental relaxation. The interval length of $20.48 \mathrm{~s}$ was derived from the algorithm for analysis of dimensional complexity which is based on 2048 data points. Smaller ocular artifacts were corrected by a regression method using the EOG as reference. The dimensional complexity (estimated correlation dimension) was calculated by combining the singular-value decomposition technique with the averaged-pointwise dimension procedure (Mölle et al, 1999; Pritchard and Duke, 1995). Mean values of dimensional complexity were computed across the three values obtained for each of the three conditions of mental activity separately for each electrode site.

In addition, log power spectra were calculated from the EEG traces using fast Fourier transformation (FFT). From the spectra, power values for the $\delta(1.0-3.5 \mathrm{~Hz}), \theta$ (3.5$7.0 \mathrm{~Hz}), \alpha(8.0-12.1 \mathrm{~Hz})$, and $\beta(14.1-30.1 \mathrm{~Hz})$ bands were obtained. Mean power values were determined across the three values for each task condition separately for each electrode site.

The answers to the convergent and divergent thinking tasks were scored by two independent and experienced raters with an inter-rater agreement of greater than $90 \%$. An answer to a task of convergent thinking was scored +3 if it was completely correct. Less points were given for solutions that were only partially correct (ie regarding subproblems). Accordingly, a maximum score of +9 could be achieved on the three tasks of a session. The answers to the divergent thinking tasks were scored according to standard criteria (Mainberger, 1977). For each task the total number of different responses (across the three epochs of thinking) was determined. Responses were excluded if they were synonymous, and if they were obviously not related to the task. If several responses occurred reflecting the same idea, only one variation of the idea was scored.

Serum concentrations of estrogen, testosterone, FSH, and LH were measured by commercially available radioimmunoassays and enzyme-linked immunoassays, respectively (estrogen, testosterone: DPC Biermann GmbH, Bad Nauheim, Germany; FSH, LH: Boehringer, Mannheim, Germany). The sensitivities were $1.4 \mathrm{pg} / \mathrm{ml}$ for estrogen determination, $4 \mathrm{ng} / \mathrm{ml}$ for testosterone and $0.5 \mathrm{mlU} / \mathrm{ml}$ for FSH and LH determination. Intraassay coefficients of variation were, respectively, $<5 \%$ (estrogen), $<8 \%$ (testosterone), $<3.8 \%(\mathrm{FSH})$, and $<3.2 \%(\mathrm{LH})$. All samples from an individual woman were analyzed in duplicate in the same assay.

\section{Statistical Analysis}

The measures of EEG dimensional complexity and power within the four frequency bands were each separately submitted to a three-way ANOVA with repeated measures representing the Treatment condition (hormone $v s$ placebo), the Tasks (divergent thinking, convergent thinking, relaxation), and the Topography $(\mathrm{F} 3, \mathrm{Fz}, \mathrm{F} 4, \mathrm{C} 3, \mathrm{Cz}, \mathrm{C} 4, \mathrm{P} 3$, $\mathrm{Pz}, \mathrm{P} 4, \mathrm{O} 1, \mathrm{O} 2)$. In pairwise 'within-contrasts', main effects and interactions concerning the Task and Topography factors were specified. To assess possible practice effects across sessions, in some additional ANOVA another factor representing the 'Order' of treatments for a subject was included. However, except for an improvement on the planning test across sessions in Experiment $\mathrm{I}(p<0.01)$, this analysis did not reveal significant effects. Analyses were done with the BMDPV4 software. Where appropriate, Greenhouse-Geisser corrected degrees of freedom were applied. Differences between the effects of the treatments on behavioral data were assessed by Wilcoxon's tests. A $p$-value of $<0.05$ was considered to be significant.

\section{RESULTS}

\section{Experiment I-Estrogen}

Transdermal estrogen treatment increased plasma estrogen concentrations more than 10 -fold over placebo values $(127.6 \pm 12.7$ vs $10.74 \pm 0.4 \mathrm{pg} / \mathrm{ml}, p<0.01)$. The concentration of $\mathrm{LH}(26.9 \pm 1.7$ vs $28.6 \pm 1.7 \mathrm{mlU} / \mathrm{ml}$ after placebo) was not affected. FSH plasma levels were lower after estrogen than placebo $(74.9 \pm 6.0$ vs $99.3 \pm 7.0 \mathrm{mlU} /$ $\mathrm{ml}, p<0.01)$.

EEG activity during thinking. Dimensional complexity of the EEG was distinctly higher during divergent than convergent thinking, while dimensionality during mental relaxation was comparable with that during divergent thinking. These differences were more pronounced over frontal than posterior cortical regions $(\mathrm{F}(20,220)=7.34$, $p<0.01$, for Task $\star$ Topography; for pairwise comparisons between divergent and convergent thinking: $\mathrm{F}(10,110)=3.34, p<0.05)$. Treatment with estrogen overall reduced EEG dimensionality $(6.06 \pm 0.26$ vs $6.36 \pm 0.29$ following placebo, $\mathrm{F}(1,11)=5.38 ; p<0.05$, for main effect of Treatment). Separate analyses on each of the three conditions of mental activity confirmed a reducing effect of estrogen on EEG dimensional complexity during divergent thinking, which showed a slight dominance over right posterior cortical regions $(\mathrm{F}(32,352)=2.7, p<0.05$, for Treatment $*$ Topography; for contrasts at P4, O2: $p<0.01$; at Fz, F3, Cz, P3, O1: $p<0.1$; Figure 1, left part). The estrogeninduced reduction in EEG dimensionality approached significance also during mental relaxation $(\mathrm{F}(1,11)=3.58$, $p<0.08$ ), but remained nonsignificant during convergent thinking.

Power spectral analysis revealed treatment-related changes for $\delta$ and $\theta$ activity. In general, $\delta$ activity was comparable during divergent and convergent thinking but reduced during mental relaxation $(\mathrm{F}(2,22)=8.53, p<0.01$; $\mathrm{F}(1,11)=4.85, p<0.05$ and $12.40, p<0.01$, for respective pairwise comparisons of mental relaxation with divergent and convergent thinking). Estrogen treatment overall enhanced $\delta$ activity (log power $9.47 \pm 0.58$ vs $9.02 \pm 0.50$ during placebo, $\mathrm{F}(1,11)=4.94, p<0.05$, Figure 2$)$, and this effect was discernible also in separate analyses of the divergent thinking and mental relaxation conditions $(\mathrm{F}(1,11)=5.46, p<0.05$ and $4.34, p<0.06$, respectively). $\theta$ activity did not discriminate divergent and convergent thinking, but was decreased over the anterior cortex during mental relaxation $(\mathrm{F}(20,220)=3.47, p<0.01$, for Task $*$ Topography). Estrogen tended to enhance $\theta$ activity $(6.74 \pm 0.63$ vs $6.16 \pm 0.68$ during placebo $(\mathrm{F}(1,11)=2.81$, $p<0.1)$ and this effect approached significance also in a separate analysis of divergent thinking $(\mathrm{F}(1,11)=3.84$, 

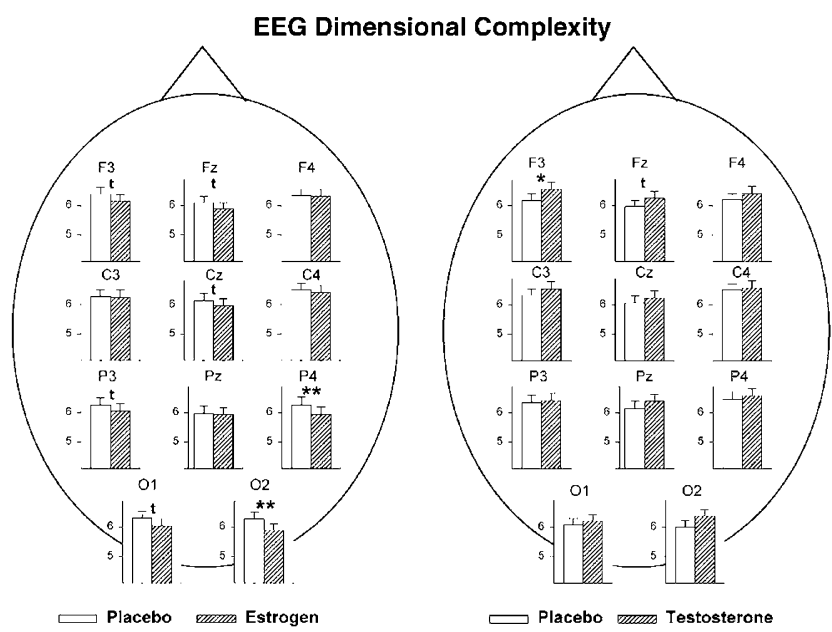

Figure I Mean ( \pm SEM) dimensional complexity of EEG activity in two groups of 12 women while solving tasks of divergent thinking. Left: women were treated transdermally with estrogen (hatched bars) and placebo (empty bars). Right: women were treated transdermally with testosterone (hatched) and placebo (empty). EEG activity was recorded from frontal (F3, $\mathrm{Fz}, \mathrm{F} 4)$, central $(\mathrm{C} 3, \mathrm{Cz}, \mathrm{C} 4)$, parietal (P3, Pz, P4), and occipital (OI, O2) electrode locations. ${ }^{*} * x<0.01,{ }^{*} p<0.05, t-p<0.1$, for pairwise comparisons between the effects of the two treatments, without adjustment for multiple comparisons.

$p<0.07) . \quad \alpha$ activity was, as expected, lower during convergent than divergent thinking or mental relaxation $(\mathrm{F}(2,20)=3.18, p<0.05)$, but was not affected by estrogen. $\beta$ power was also smaller during convergent than divergent thinking particularly at the central and parietal leads $(\mathrm{F}(20,220)=4.89, p<0.01$, for Task $*$ Topography $)$, and was also not affected by estrogen treatment.

Performance measures of thinking, motor perseveration, memory, and mood. On tests of divergent thinking, the women performed worse following estrogen than placebo. This difference reached significance for the 'Consequences' subtest, when EEG activity was concurrently recorded, as well as for the 'Instances' subtest $(\mathrm{F}(1,11)=4.65$ and 5.15 , respectively, $p<0.05$, Table 1 ). Also, the overall score of divergent thinking determined by the average score across all six subtests was lower after estrogen than placebo treatment $\quad(8.27 \pm 0.57 \quad v s \quad 9.34 \pm 0.78, \quad F(1,11)=15.72$, $p<0.01$, Table 1). In contrast, performance on convergent thinking tasks was better following estrogen than placebo (mean \pm SEM score $7.53 \pm 0.99$ vs $5.46 \pm 0.68, \mathrm{~F}(1,11)=$ $6.80, p<0.05)$. Consistent with the inferior divergent thinking performance, subjects following estrogen also displayed a stronger tendency to perseverate on the test of motor perseveration $(\mathrm{F}(1,11)=5.68, p<0.05$, Table 2$)$.

Analysis of word recall across both lists did not reveal significant differences between the effects of estrogen and placebo. However, separate analysis on each word list (summarized in Table 2) indicated superior recall after estrogen than placebo for the first list $(F(1,11)=6.63$, $p<0.05)$ while performance on the second list was comparable for both conditions $(\mathrm{F}(1,11)=0.27$, n.s. $)$. There was no effect of estrogen on self-reported mood and activation.

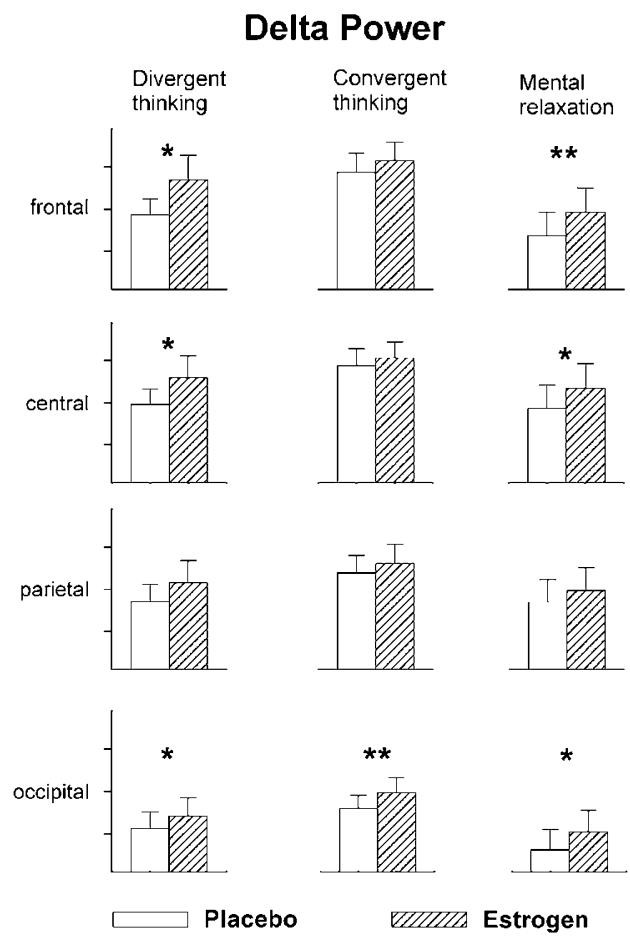

Figure 2 Mean ( \pm SEM) log power (arbitrary units) in the $\delta$ frequency band in 12 postmenopausal women tested following administration of placebo (empty bars) and estrogen (hatched bars) during solving tasks of divergent and convergent thinking and during mental relaxation. Data are averaged, respectively, across the frontal, central, parietal, and occipital recording sites. ${ }^{*} * p<0.01$, ${ }^{*} p<0.05$, for pairwise comparisons between the effects of estrogen and placebo.

Table I Divergent Thinking Following Estrogen and Placebo Treatment

\begin{tabular}{lcrl}
\hline Subtest & Estrogen & Placebo & \\
\hline Consequences & $7.62 \pm 0.71$ & $8.71 \pm 0.94$ & $p<0.05$ \\
Planning & $9.21 \pm 1.08$ & $10.01 \pm 0.96$ & \\
Pattern meaning & $7.30 \pm 0.66$ & $7.46 \pm 0.73$ & \\
Instances & $9.46 \pm 1.11$ & $12.07 \pm 1.19$ & $p<0.05$ \\
Alternate uses & $8.53 \pm 0.86$ & $9.15 \pm 0.93$ & \\
Synonyms & $7.53 \pm 0.62$ & $8.69 \pm 0.89$ & \\
Overall score & $8.27 \pm 0.57$ & $9.34 \pm 0.78$ & $p<0.01$ \\
\hline
\end{tabular}

Mean $( \pm$ SEM) score for six subtests and the overall score of divergent thinking are shown, $n=12$. The right column indicates significance between the effects of the treatments.

\section{Experiment II-Testosterone}

Transdermal testosterone treatment increased plasma testosterone concentrations to values comparable with concentrations during the ovulatory phase in young women (testosterone: $0.57 \pm 0.07 \mathrm{ng} / \mathrm{ml}$; placebo: $0.19 \pm 0.02 \mathrm{ng} / \mathrm{ml}$, $p<0.01)$. Concentrations of estrogen $(17.22 \pm 9.0 v s 11.23$ $\pm 4.5 \mathrm{pg} / \mathrm{ml}$, after placebo), FSH $(79.52 \pm 8.9$ vs $82.76 \pm$ $5.4 \mathrm{mlU} / \mathrm{ml})$, and $\mathrm{LH}(29.11 \pm 3.8$ vs $31.73 \pm 3.3 \mathrm{mlU} / \mathrm{ml})$ did not differ between conditions.

EEG activity during thinking. Analysis of dimensional complexity of the EEG confirmed, as in Experiment I, a generally enhanced complexity during solving tasks of 
Table 2 Convergent Thinking, Motor Perseveration, and Verbal Memory Following Estrogen and Placebo

\begin{tabular}{lccc}
\hline Test & Estrogen & Placebo & \\
\hline Convergent thinking & $7.53 \pm 0.99$ & $5.46 \pm 0.68$ & $p<0.05$ \\
Motor perseveration & $1.20 \pm 0.02$ & $1.13 \pm 0.01$ & $p<0.05$ \\
Verbal memory & $7.6 \pm 0.56$ & $6.2 \pm 0.46$ & $p<0.05$ \\
$\quad$ Ist word list & $6.3 \pm 0.53$ & $6.6 \pm 0.52$ & \\
2nd word list &
\end{tabular}

Mean ( \pm SEM) scores on tests of convergent thinking, motor perseveration, and verbal memory. The score of convergent thinking is summed across three tasks. A completely correct solution of a task was scored ' 3 '. Motor perseveration scores refer to the reciprocal of the entropy values characterizing the sequence of button press responses, which according to the subject's instruction should be as 'chaotic' as possible. Verbal memory was tested by immediate recall of two lists of 15 words, $n=12$. The right column indicates significance between the effects of the treatments.

divergent than convergent thinking, with this difference again being most pronounced over frontal cortical regions $(\mathrm{F}(20,220)=4.31, p<0.01$, for Task $\star$ Topography; for pairwise comparison between divergent and convergent thinking: $(\mathrm{F}(10,110)=3.64, p<0.01)$. While there was no effect of testosterone on EEG dimensional complexity in the overall analysis, separate analysis of the three mental conditions revealed a tendency of testosterone to increase EEG complexity during divergent thinking $(F(1,11)=3.10$, $p<0.1$ ), which in comparisons at single electrode sites reached significance at F3 (Figure 1).

Analyses of EEG power spectra also confirmed the main difference found between the conditions of divergent and convergent thinking in Experiment I. $\delta$ and $\theta$ activity again were higher during both conditions of thinking than during mental relaxation $(\mathrm{F}(2,22)=17.24$ and 9.87, $p<0.01$, respectively). $\alpha$ and $\beta$ activity were, over the posterior association cortex, higher during divergent than convergent thinking $(\mathrm{F}(20,220)=3.00$ and $3.75, p<0.01$ for respective Task $*$ Topography interactions). However, testosterone did not induce any significant changes in the different frequency bands.

Performance measures of thinking, motor perseveration, memory, and mood. Performance on tasks of divergent creative thinking overall tended to be improved after testosterone as compared to the effects of placebo $(\mathrm{F}(1,11)=3.80, p<0.07$, Table 3). Differences in the same direction between the treatment conditions were revealed to be significant for the 'Consequences' subtest $(\mathrm{F}(1,11)=4.71$, $p<0.05)$ and for the 'Alternate Uses' subtest $(\mathrm{F}(1,11)=5.06$, $p<0.05)$ and approached significance for the 'Synonyms' subtest $(F(1,11)=4.11, p<.06)$. Notably, a supplementary analysis performed to explore the relation between the increases in overall divergent thinking and plasma testosterone concentration revealed a negative correlation $(r=-0.82, p<0.01)$, that is, the women with only slight increases in testosterone concentration had the most distinct increases in divergent thinking. Performance on tasks of convergent thinking (mean \pm SEM score, testosterone: $3.50 \pm 0.39$; placebo: $4.16 \pm 0.71, \quad p>0.4$ ), motor perseveration (testosterone: $1.12 \pm 0.02$; placebo: $1.11 \pm 0.01, p>0.4)$, and the average number of recalled words (across both lists, testosterone: $7.6 \pm 0.40$; placebo:
Table 3 Divergent Thinking Following Testosterone and Placebo Treatment

\begin{tabular}{lrcl}
\hline Subtest & Testosterone & Placebo & \\
\hline Consequences & $8.63 \pm 0.89$ & $6.81 \pm 0.60$ & $p<0.05$ \\
Planning & $11.27 \pm 1.02$ & $9.81 \pm 0.50$ & \\
Pattern meaning & $6.81 \pm 0.55$ & $7.63 \pm 0.65$ & \\
Instances & $8.19 \pm 0.89$ & $8.65 \pm 0.99$ & \\
Alternate uses & $10.54 \pm 1.06$ & $8.54 \pm 0.99$ & $p<0.05$ \\
Synonyms & $7.54 \pm 0.73$ & $6.54 \pm 0.72$ & $p<0.07$ \\
Overall score & $8.91 \pm 0.58$ & $8.01 \pm 0.53$ & $p<0.07$ \\
\hline
\end{tabular}

See Table I.

$6.9 \pm 0.41, p>0.3)$ did not differ between treatment conditions. Also, self-reported mood did not indicate distinct influences of testosterone.

\section{DISCUSSION}

This study indicates effects of estrogen and testosterone on working memory functions and EEG activity in older women already following a treatment period of only 3 days. The data thereby add to a growing body of evidence for gonadal steroid effects on female cognition, derived mainly from studies of prolonged hormonal replacement therapy. Our data show that estrogen treatment impairs abilities of divergent creative thinking and reduces in parallel dimensional complexity of EEG activity. In contrast, convergent, analytical thought and verbal memory were enhanced following estrogen. Effects of testosterone appeared to be less consistent, and for the main variables opposite to those of estrogen.

Regarding estrogen, several previous studies consistently indicated a favorable effect of estrogen replacement treatment on verbal short-term memory functions (for reviews, see LeBlanc et al, 2001; Sherwin, 2002). Thus, recall of materials encoded into working memory shortly before was found to be improved in menopausal and postmenopausal women treated with estrogen (eg Rice et al, 1997; Verghese et al, 2000). The women of the present study showed likewise increased recall of words from the first list. That this effect did not extend to the second list presented immediately after the first could reflect a type of enhanced proactive interference after superior encoding of the first word list. Yet, this view needs to be further substantiated. Collectively, these findings may speak for an enhanced encoding of stimuli into working memory following estrogen, which facilitates their later recall but may also interfere with effective encoding of succeeding stimuli.

Improved verbal memory following estrogen treatment could be viewed to stem from a more basic shift in the mode of working memory processing, as reflected by the opposite effects of estrogen on divergent and convergent thinking. Compared with convergent thinking, divergent thinking is assumed to rely on an enhanced spread of activation in cortical networks so that multiple representations in working memory become fluently activated. Accordingly, the greater dimensional complexity of EEG activity during divergent thinking has been linked to a greater number of cortical neuronal assemblies (ie representational units of 
thoughts and ideas) concurrently activated (Mölle et al, 1996, 1999). Conversely, lowered EEG complexity during convergent thinking (typically associated with increased $\delta$ activity) has been linked to the prominent feature of this mode of thinking, that is, to focus on the only and one right answer to a given problem, thereby suppressing any irrelevant neuronal assembly activity. On this background, signs of improved convergent and impaired divergent thinking following estrogen indicate a shift in the mode of working memory processing from a widespread activation of weakly associated representational units to the focused activation of units with strongly interconnected associative links. That the reduction in EEG dimensionality following estrogen showed a slight predominance over the right posterior cortex during divergent thinking in this context could reflect characteristics of the task stimulating visual imagery. The view of an estrogen-induced shift towards increased convergent processing would also be consonant with enhanced memory encoding and explain the more stereotyped behavior on the motor perseveration test. Moreover, this shift appears to be in line with previous observations of enhanced abstract reasoning and verbal memory in older women with higher ( $v s$ lower) basal estrogen levels and during replacement therapy, respectively (Rice et al, 1997; Drake et al, 2000; LeBlanc et al, 2001). Results regarding verbal fluency were inconclusive in those studies (eg Drake et al, 2000; Grodstein et al, 2000).

The brain regions mediating the effect of estrogen on thinking are not known. The regulation of search and retrieval processes underlying thinking presumably involves a distributed network of regions including prefrontal cortical and limbic structures (eg Miller, 2000; Fuster, 2000). Two estrogen receptor subtypes are known, which are predominantly expressed in limbic regions including the amygdala (mainly the $\alpha$ subtype) and the hippocampal formation (mainly the $\beta$ subtype), but are also expressed in the neocortex (Osterlund and Hurd, 2001). Estrogen could exert its influence by increasing inhibitory GABAergic activity in such networks (eg Rudick and Woolley, 2001).

Our findings regarding testosterone point towards an enhanced divergent mode of processing. They appear to be consonant with the few previous reports that related effects of testosterone to female cognition, indicating associations of high endogenous testosterone with musical creativity in younger women (Hassler, 1991, 1992) and with verbal fluency in postmenopausal women (Drake et al, 2000; Barrett-Connor and Goodman-Gruen, 1999). Also, in oophorectomized women, testosterone treatment improved various measures of cognitive abilities, including verbal fluency (Sherwin, 1988). The effects of testosterone on divergent thinking here were opposite to those of estrogen, which argues against the possibility that testosterone acts, after tissue specific aromatization, mainly via central nervous estrogen receptors (eg Balthazart et al, 2001). Nevertheless, such conversion may occur to a certain extent, which could explain why the effects of testosterone were overall weaker than those of estrogen, and also that the increase in divergent thinking was only relatively small in women showing the greatest increase in plasma testosterone concentration after this treatment. Androgen receptors that could mediate cognitive effects of testosterone have been identified in the hippocampus and the parietal cortex, although receptor expression in these regions is distinctly weaker than in the hypothalamus and amygdala showing highest androgen receptor density (Abdelgadir et al, 1999; Beyenburg et al, 2000).

The present experiments should be considered a pilot study given the small number of women examined with multiple tests. Thus, it must be cautioned against overgeneralizing our findings, although the significance of changes indicates that in older women gonadal steroids can exert quite distinct actions on female cognition even pertaining to behaviors with relevance for everyday life. Also, despite the fact that the induced changes in plasma levels of estrogen and testosterone were comparable with those naturally occurring around ovulation in spontaneously cycling women, it remains highly questionable as to whether our results can be transferred to the conditions in young women, where estrogen and testosterone act in a specifically timed concert with multiple other gonadal hormones. Unlike young women, older women display continuously enhanced levels of FSH and $\mathrm{LH}$, and a decline in abilities of thinking, more pronounced for divergent than convergent thought (Andersson et al, 1978; McCrae et al, 1987; Costa and McCrae, 1993). In addition, EEG dimensional complexity increases with age (Anokhin et al, 1996). All these age-dependent alterations in endocrine and neurocognitive functions may explain why despite enhanced estrogen concentration around ovulation, young women display enhanced divergent thinking, while postmenopausal women respond to similar increases in estrogen with enhanced convergent thinking.

\section{ACKNOWLEDGEMENTS}

We thank C Zinke, K Brooks, and T Nelskamp for their excellent technical assistance. This work was supported by a grant from the Deutsche Forschungsgemeinschaft to JB. The authors declare that they have no competing financial interests.

\section{REFERENCES}

Abdelgadir SE, Roselli CE, Choate JV, Resko JA (1999). Androgen receptor messenger ribonucleic acid in brains and pituitaries of male rhesus monkeys: studies on distribution, hormonal control, and relationship to luteinizing hormone secretion. Biol Reprod 60: 1251-1256.

Andersson E, Berg S, Lawenius M, Svanborg A (1978). Intellectual functioning in a 70-years-old urban population. Acta Psychiatr Scand 57: 59-66.

Anokhin AP, Birbaumer N, Lutzenberger W, Nikolaev A, Vogel F (1996). Age increases brain complexity. Electroencephalogr Clin Neurophysiol 99: 63-68.

Balthazart J, Bauillien M, Ball GF (2001). Phosphorylation processes mediate rapid changes of brain aromatase activity. $J$ Steroid Biochem Mol Biol 79: 261-277.

Barrett-Connor E, Goodman-Gruen D (1999). Cognitive function and endogenous sex hormones in older women. J Am Geriatr Soc 47: 1289-1293.

Beyenburg S, Watzka M, Clusmann H, Blumcke I, Bidlingmaier F, Elger CE et al (2000). Androgen receptor with mRNA expression in the human hippocampus. Neurosci Lett 294: 25-28.

Bollinger G (1981). Kreativitätsmessung durch Tests zum divergenten Denken? Z Dif Diagn Psychol 2: 87-106. 
Brunel U (1991). Cognition, memory and the menstrual cycle. Cah Psychol Cognit 11: 3-26.

Costa Jr PT, McCrae RR (1993). Psychological research in the Baltimore Longitudinal Study of Aging. Z Gerontol 26: 138-141.

Drake EB, Henderson VW, Stanczyk FZ, McCleary CA, Brown WS, Smith CA et al (2000). Associations between circulating sex steroid hormones and cognition in normal elderly women. Neurology 54: 599-603.

Fuster JM (2000). Memory networks in the prefrontal cortex. Prog Brain Res 122: 309-316.

Grodstein F, Chen J, Pollen DA, Albert MS, Wilson RS, Folstein MF et al (2000). Postmenopausal hormone therapy and cognitive function in healthy older women. J Am Geriatr Soc 48: 746-752.

Guilford JP (1967). The Nature of Human Intelligence. McGrawHill: New York.

Guilford JP, Hoepfner R (1971). The Analysis of Intelligence. McGraw-Hill: New York.

Hampson E (1990). Estrogen related variations in human spatial and articulatory-motor skills. Psychoneuroendocrinology 15: 97111.

Hassler M (1991). Testosterone and musical talent. Exp Clin Endocrinol 98: 89-98.

Hassler M (1992). Creative musical behavior and sex hormones: musical talent and spatial ability in the two sexes. Psychoneuroendocrinology 17: 55-70.

Hausmann M, Slabbekoorn D, Van Goozen SH, Cohen-Kettenis PT, Gunturkun O (2000). Sex hormones affect spatial abilities during the menstrual cycle. Behav Neurosci 114: 1245-1250.

Janke W, Debus G (1978). Die Eigenschaftswörterliste EWL. Eine mehrdimensionale Methode zur Beschreibung von Aspekten des Befindens. Hogrefe: Göttingen.

Krug R, Finn M, Pietrowsky R, Fehm HL, Born J (1996). Jealousy, general creativity, and coping with social frustration during the menstrual cycle. Arch Sex Behav 24: 181-199.

Krug R, Mölle M, Fehm HL, Born J (1999). Variations across the menstrual cycle in EEG activity during thinking and mental relaxation. J Psychophysiol 13: 163-172.

Krug R, Stamm U, Pietrowsky R, Fehm HL, Born J (1994). Effects of menstrual cycle on creativity. Psychoneuroendocrinology 19: $21-31$.

LeBlanc ES, Janowsky J, Chan BK, Nelson HD (2001). Hormone replacement therapy and cognition: systematic review and metaanalysis. JAMA 285: 1489-1499.

Lutzenberger W, Preissl H, Pulvermüller F (1996). Fractal dimension of electroencephalographic time series and underlying brain processes. Biol Cybernet 73: 477-482.

Mainberger U (1977). Tests zum divergenten Denken (Kreativität) TDK 4-6. Beltz: Weinheim.

McCrae RR, Arenberg D, Costa Jr PT (1987). Declines in divergent thinking with age: cross-sectional, longitudinal, and crosssequential analyses. Psychol Aging 2: 130-137.
Miller EK (2000). The prefrontal cortex and cognitive control. Nat Rev Neurosci 1: 59-65.

Mittenecker E (1960). Die informationstheoretische Auswertung des Zeigeversuchs bei Psychotikern und Neurotikern. Z Exp Angew Psychol 7: 392-400.

Mölle M, Marshall L, Lutzenberger W, Pietrowsky R, Fehm HL, Born J (1996). Enhanced dynamic complexity in the human EEG during creative thinking. Neurosci Lett 208: 61-64.

Mölle M, Marshall L, Wolf B, Fehm HL, Born J (1999). EEG complexity and performance measures of creative thinking. Psychophysiology 36: 95-104.

Osterlund MK, Hurd YL (2001). Estrogen receptors in the human forebrain and the relation to neuropsychiatric disorders. Prog Neurobiol 64: 251-267.

Pritchard WS, Duke D (1995). Measuring "chaos" in the brain: a tutorial review of EEG dimension estimation. Brain Cogn 27: 353-397.

Regestein QR, Friebely J, Shifren J, Schiff I (2001). Neuropsychological effects of methyltestosterone in women using menopausal hormone replacement. J Womens Health Gend Based Med 10: 671-676.

Rice MM, Graves AB, McCurry SM, Larson EB (1997). Estrogen replacement therapy and cognitive function in postmenopausal women without dementia. Am J Med 103: 26-35.

Rudick CN, Woolley CS (2001). Estrogen regulates functional inhibition of hippocampal CA1 pyramidal cells in the adult female rat. J Neurosci 21: 6532-6543.

Schwibbe MH (1985). Experimentelle Untersuchungen zur Eignung des Mittenecker-Zeigeversuchs für klinische Langzeitstudien und zur Validität seiner Testparameter. Z Klin Psychol 14: $130-144$.

Sherwin BB (1988). Estrogen and/or androgen replacement therapy and cognitive functioning in surgically menopausal women. Psychoneuroendocrinology 13: 345-357.

Sherwin BB (1999). Can estrogen keep you smart? Evidence from clinical studies. J Psychiatr Neurosci 24: 315-321.

Sherwin BB (2002). Estrogen and cognitive aging in women. Trends Pharmacol Sci 23: 527-534.

Slob AK, Bax CM, Hop WC, Rowland DL, van der Werff ten Bosch JJ (1996). Sexual arousability and the menstrual cycle. Psychoneuroendocrinology 21: 545-558.

Verghese J, Kuslansky G, Katz MJ, Sliwinski M, Crystal HA, Buschke $\mathrm{H}$ et al (2000). Cognitive performance in surgically menopausal women on estrogen. Neurology 55: 872-874.

Wallach MA, Kogan N (1969). Modes of thinking in young children. Holt, Rinehart \& Winston: New York.

Wechsler D (1981). Wechsler Adult Intelligence Scale-Revised (WAIS-R). Psychological Corporation: New York.

Wolf OT, Kirschbaum C (2002). Endogenous estradiol and testosterone levels are associated with cognitive performance in older women and men. Horm Behav 41: 259-266. 